\title{
Latent Class Approach to Estimate the Willingness to Pay for Transit User Information
}

\author{
Pietro Zito*, Giuseppe Salvo \\ Department of Energy_-Transportation Group, University of Palermo, Palermo, Italy \\ Email: " pietro.zito@unipa.it
}

Received April 8, 2012; revised May 2, 2012; accepted May 28, 2012

\begin{abstract}
The aim of analysis is to understand how unreliable information influences user behaviour and how much it discourages public transport use. For this purpose, a Stated Preference Survey was carried out in order to know the preferences of public transport users relating to information needs and uncertainty on the information provided by Advanced Traveller Information System (ATIS). The perceived uncertainty is defined as information inaccuracy. In our study, we considered the difference between forecasted or scheduled waiting time at the bus stop and/or metro station provided by ATIS, and that experienced by user, to catch the bus and/or metro. A questionnaire was submitted to an appropriate sample of Palermo's population. A Latent Class Logit model was calibrated, taking into account attributes of cost, information inaccuracy, travel time, waiting time, and cut-offs in order to reveal preference heterogeneity in the perceived information. The calibrated model showed various sources of preference heterogeneity in the perceived information of public transport users as highlighted by the analysis reported. Finally, the willingness to pay was estimated, confirming a great sensitivity to the perceived information, provided by ATIS.
\end{abstract}

Keywords: Preference Heterogeneity; Latent Class Model; Perceived Information; Uncertainty; Willingness to Pay

\section{Introduction}

The Advanced Traveller Information Systems (ATIS) includes a broad range of advanced computer and communication technologies. These systems are designed to provide transit riders pre-trip and real-time information, so as to make better informed decisions regarding their mode of travel, planned routes, and travel times. ATIS's include in-vehicle devices, terminal or wayside based information centres, information by phone or mobile, and internet.

There is a substantial literature concerning the user behaviour in relation to information provided by ATIS, distinguishing the following [1]:

- On one side, the viewpoint of marketing concerning the potential of ATIS as a business case, either stand alone or as part of an effort to gain or retain users for urban transit [2-6];

- On other side, the viewpoint of ATIS as a potential tool for Travel Demand Management (TDM), [7-13], who investigate the expectations of travel information provision as a means to change traveler behavior as the modal shift from private car to transit;

- Finally, the viewpoint of individuals, when these face with choice-situations under uncertainty, they can

*Corresponding author. make mistakes since travel choices often involve uncertainty on travel time, route choice, scheduled waiting time and so on [14-18].

The paper focuses on some issues relating to how transit users may be uncertain about how to perceive the information when they are unreliable and affected by error or uncertainty.

Abdel-Aty et al. [2], studied the effects of ATIS on route choice by stated preference analysis observing a consequent reduction in travel time uncertainty. Also, Abdel-Aty et al. [3], studied the commuter propensity to use transit with a computer-aided telephone interview conducted in Sacramento and San Jose, California. The results indicated that approximately $38 \%$ of the respondents who currently do not use transit might consider public transport if the appropriate information is available. Moreover, using an ordered probit model produced results that show the significant effect of several commute and socioeconomic characteristics on the propensity to use public transport.

Recently, Molin and Timmermans [5] evaluated the willingness to pay for additional information through web enabled public transport information systems. Dziekan and Kottenhoff [19], showed the main effects of the ATIS: reduced wait time, positive psychological factors, such as reduced uncertainty, simplified use and a greater 
feeling of security, increased willingness to pay, adjusted travel behaviour, such as better use of wait time or more efficient travelling, mode choice effects, higher customer satisfaction and better image.

Polak and Jones [20], under the DRIVE European Project, studied the effects of pre-trip information on travel behaviour using a stated preference approach in Birmingham and Athens. The analysis revealed firstly that there was requirement for multimodal pre-trip travel information although the sample studied was made up of regular car users, and that the quantity and type of pretrip information requested by travellers depends on a range of personal, journey related, contextual and national factors. Moreover, they emphasised the importance to travellers of the timeliness and relevance of the provided information especially when relevant network incidents happen.

Nijkamp et al. [21] conducted a survey before and after the application of ATIS in the city of Birmingham and Southampton (QUARTET and STOPWATCH project respectively). Due to the small sample examined in the QUARTET project their result was considered unreliable, whereas in the city of Southampton the survey revealed a rise in using public transport, especially, in study and leisure trips, and mobility optimisation of people in choosing the mode and route able to reduce travel time. A methodology was developed by Mishalani et al. [22], aiming to understand the effect of real-time information on bus stops, under three different methods to forecast bus stop arrival time: 1) static information, 2) real-time information up-date using historical data, 3) real-time information using data coming from an Automatic Vehicle Location (AVL) system. Measures of the difference between predicted and effective waiting time when people approach a bus stop showed that the third method revealed to be more reliable than the other two methods.

Several authors analysed the commuters' behaviour under ATIS environment, in particular travel time and route choice, such as [23]. Grotenhuis et al. [24] investigated the desired quality of integrated multimodal travel information in public transport. Polydoropoulou and Ben-Akiva [6], Chorus et al. [16], Lappin [25] showed that perception of information can be explained by behavioural factors. Furthermore, Chien et al. [26] and Tan et al. [27] set up decision support systems: the former to provide real-time pre-trip information on bus arrival times; whereas the latter to find a reasonable path in transit networks validated by a survey.

The impacts of benefits and technical performance of communication technology application in the city of Helsinki was studied by Lehtonen and Kulmala [28]. The system provided several public transport telematics, such as real-time passenger information, bus and tram priori- ties at traffic signals and schedule monitoring. Before and after field studies, an interview and survey, a simulation and socioeconomic evaluation indicated a $40 \%$ reduction of delay at signals, improving on regularity and reliability of public transport, and reductions of $1 \%-5 \%$ in fuel consumption and exhaust emissions. Moreover, the information systems were regarded very positively, and, in particular the information displays at stops were considered necessary. Similarly, Luk and Yang [29] showed the benefits of ATIS application in Singapore. Travel information may play a central role in reducing uncertainty influencing the transport demand [30] and/or reducing the perceived waiting time [31].

Some studies have pointed out as individuals, when face with choice-situations in a state of uncertainty, can make mistakes since travel choices often involve uncertainty on travel time, route choice, scheduled waiting time and so on [14-18]. In particular, Chorus et al. [16] discussed travellers' need for personalised and more advanced types of travel information.

The paper focuses on some issues relating to how transit users may be uncertain about how to perceive the information when they are unreliable and affected by error or uncertainty. The main innovative task of analysis is to understand how unreliable information influences user behaviour and how much it discourages public transport use. For this purpose, a stated preference survey was run by submitting a questionnaire to a sample of population of Palermo, in order to know preferences of public transport users, information user needs and how unreliable information provided by ATIS influences user behaviour.

We consider two competing alternatives, namely private car and public transport; distinguishing between cardrivers and transit-users and therefore are interested to evaluate the reaction of both users categories to the information provided by ATIS for public transport.

The perceived uncertainty is defined as the information inaccuracy. In our study, we considered the differrence between forecasted or scheduled waiting time at the bus stop and/or metro station provided by ATIS, and that experienced by users, who want to catch the bus and/or metro.

Furthermore, another original aspect regards the preference heterogeneity in the information perceived by public transport users, identifying in the decision process the unobserved heterogeneity sources. The presence of preference heterogeneity in the interviewed population sample allows one better to explain the underlying individual choice mechanisms. For this task, a latent class model was calibrated, taking into account attributes of cost, information inaccuracy, travel time, waiting time, and their cut-offs and comparing the results with those of the traditional multinomial logit. 
The existence of cut-offs and their utilization in decision problems is widely recognized. The decision maker has limited ability to collect and process information. Therefore he/she chooses in two stages. In the former, the decision maker chooses the best one among available alternatives, taking into account a non-compensative decision process, in which any attribute is compared with the relative threshold (cut-off). In the latter, the decision maker weights remaining alternatives by a compensative decision process considering their different attributes [32].

The paper is structured as follows: Section 2 shows the survey and user information needs; Section 3 describes the theoretical aspects of the latent class logit model; Section 4 points out the model specification; in Section 5 the outcomes are shown and critically discussed; in Section 6 the willingness to pay is estimated and finally conclusion and future steps are given.

\section{The Survey and Information User Needs}

The survey was carried out in March 2009 in Palermo. The latter is the main Sicilian city, with surface area of 158 square $\mathrm{km}$ and a population of about 700.000 inhabitants, with a large historical area (about 2.7 square $\mathrm{km})$. This area is the centre of the main directional and administrative functions of the island. Public transport by bus covers almost all areas of the city, but only a few lines run on a reserved lane (Figure 1). Thus performances are influenced by congestion of private mobility causing inefficiency in the level of service (travel and waiting time and scheduling). Furthermore, the city has few parking areas and has no interchange with other transport modes ("Park \& Ride").

In the metropolitan area, the mass rapid transit system, when completed, will be performed by a fundamental rail transport network composed by light rail, through railway and underground; and a feeder tram system with three tram lines. The realization of an integrated mass rapid transit system with interchange nodes and stations will make it possible to improve trips inside the metropolitan area, by using interchange parking areas and park $\&$ ride policy (such as Roccella parking area).

At time of analysis, no real time information was provided by Road Local Public Transport Company (AMAT), whereas it was provided for railway system and underground. The survey was conducted using a mailback self-completion questionnaire.

The first step in the design of the questionnaire was to identify the most significant attributes for our analysis, taking into account the cost, the information inaccuracy, the travel time, the waiting time at the bus stop and the terminal (Table 1).

In particular, the travel time from different origins and
Table 1. The choice scenario with levels of the attributes.

\begin{tabular}{|c|c|c|}
\hline Attribute & Private car & Transit \\
\hline Daily cost & $6 €$ & $2.60-3.20 €$ \\
\hline $\begin{array}{l}\text { Waiting time for transit/parking } \\
\text { research time for private car }\end{array}$ & $10 \min$ & $5-15 \min$ \\
\hline Information inaccuracy & - & $4-10 \min$ \\
\hline Travel time & $20-30 \mathrm{~min}$ & $25 \mathrm{~min}$ \\
\hline
\end{tabular}

destinations were estimated elaborating a D.U.E. (Deterministic User Equilibrium) process of assignment of the private car O/D matrix (related to the rush hour and the average working day) to the urban network (Comune di Palermo, 1997). Daily cost was estimated considering maintenance costs, motor vehicle tax, civil liability and the number of kilometres travelled per year, which we supposed to be equal to $15,000 \mathrm{~km}$ and a medium size car; whereas for daily costs of public transport, the ticket cost was increased of the information cost $(10$ - 30 cents of euro) estimated by a pilot survey. Waiting time and information inaccuracy were estimated by a pilot survey in order to determine the waiting time experienced and the information inaccuracy.

The full factorial design provides $k^{n}=2^{4}=16$ different scenarios (where $n$ is the number of attributes and $k$ is the number of levels). Thus, assuming the irrelevance of interactions between attributes, in accordance with the technique of Kocur et al. [33], we identified 8 different scenarios (fractional factorial design).

In the questionnaire, firstly, we asked respondents to give a value about their maximum threshold of the considered attribute (cut-offs), in order to achieve an improved public transport service through the ATIS. Cutoff information was gathered for following attributes: information cost (upper bound), the information inaccuracy (upper bound), the waiting time (upper bound).

Further, we also asked to the decision makers to select between private car and transit in eight scenarios. Also, other information was collected: frequency of use of bus and private vehicle, evaluation of the importance of some factors in choice of whether or not travel using private and public transport, some transport habits (frequency, purpose and maximum distance travelled with transport modes), information user needs and quality travel information, and some socioeconomic information, such as household income, age, gender etc. (Ortúzar, [34]).

We submitted 250 questionnaires (whose 110 correctly compiled) to a sample of citizens chosen among potential transit users (as students, employees, etc.). Furthermore, the width of interviewed sample is about $0.3 \%$, considering a universe of about 40,000 transit users per day (related to an average share of $15 \%$ in transit modal choice in Palermo, ISTAT, 2006). Table 2 provides response 

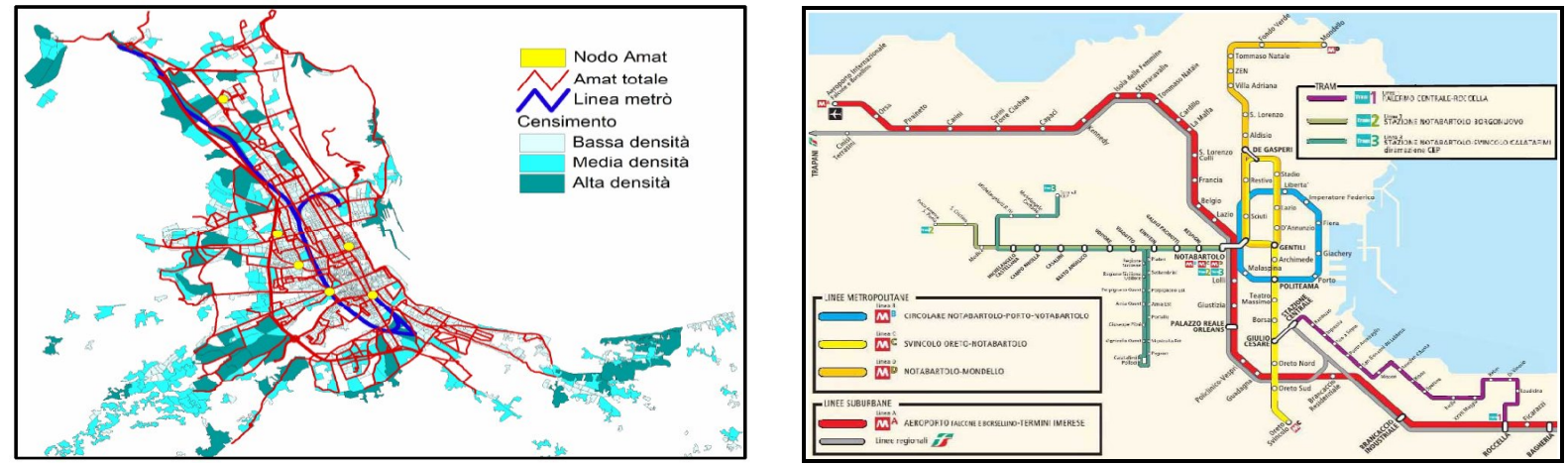

Figure 1. Road and rail public transport respectively.

Table 2. Response group characteristics $(n=110)$.

\begin{tabular}{|c|c|c|c|c|c|}
\hline Attribute & Proportion \% & Cumulative \% & Attribute & Proportion \% & Cumulative \% \\
\hline Age & & & Frequency & & \\
\hline $18-24$ & $10.10 \%$ & $10.10 \%$ & Daily & $72.73 \%$ & $72.73 \%$ \\
\hline $25-34$ & $31.31 \%$ & $41.41 \%$ & $3 / 4$ times for week & $16.16 \%$ & $88.89 \%$ \\
\hline $35-44$ & $25.25 \%$ & $66.67 \%$ & $1 / 2$ times for week & $5.05 \%$ & $93.94 \%$ \\
\hline $45-64$ & $29.29 \%$ & $95.96 \%$ & $2 / 3$ times for month & $3.03 \%$ & $96.97 \%$ \\
\hline$>65$ & $4.04 \%$ & $100.00 \%$ & Once for month & $3.03 \%$ & $100.00 \%$ \\
\hline Gender & & & Type of looked for information & & \\
\hline Male & $58.59 \%$ & $58.59 \%$ & Weather & $13.57 \%$ & $13.57 \%$ \\
\hline Female & $41.41 \%$ & $100 \%$ & Traffic cond. & $11.56 \%$ & $25.13 \%$ \\
\hline Household income & & & Route & $22.61 \%$ & $47.74 \%$ \\
\hline$<25,000 €$ & $28.28 \%$ & $28.28 \%$ & Lim. traffic zone & $11.06 \%$ & $58.79 \%$ \\
\hline $25,000-50,000 €$ & $39.39 \%$ & $67.68 \%$ & Availability of parking areas & $11.06 \%$ & $69.85 \%$ \\
\hline $50,000-75,000 €$ & $20.20 \%$ & $87.88 \%$ & Altern. modes to private car & $13.57 \%$ & $83.42 \%$ \\
\hline$>75,000 €$ & $12.12 \%$ & $100.00 \%$ & Dep./arr. time for transit & $11.56 \%$ & $94.97 \%$ \\
\hline Owned car number & & & Nothing & $5.03 \%$ & $100.00 \%$ \\
\hline 0 & $1.01 \%$ & $1.01 \%$ & Source of information & & \\
\hline 1 & $18.18 \%$ & $19.19 \%$ & Web site & $32.00 \%$ & $32.00 \%$ \\
\hline 2 & $41.41 \%$ & $60.61 \%$ & Map & $16.00 \%$ & $48.00 \%$ \\
\hline 3 & $30.30 \%$ & $90.91 \%$ & GPS & $14.00 \%$ & $62.00 \%$ \\
\hline 4 & $6.06 \%$ & $96.97 \%$ & TV/RD & $5.33 \%$ & $67.33 \%$ \\
\hline 5 & $3.03 \%$ & $100.00 \%$ & Call center & $2.00 \%$ & $69.33 \%$ \\
\hline Household number & & & Mobile phone & $4.00 \%$ & $73.33 \%$ \\
\hline 1 & $2.02 \%$ & $2.02 \%$ & E-kiosk & $1.33 \%$ & $74.67 \%$ \\
\hline 2 & $10.10 \%$ & $12.12 \%$ & News paper & $14.67 \%$ & $89.33 \%$ \\
\hline 3 & $25.25 \%$ & $37.37 \%$ & Nothing & $10.67 \%$ & $100.00 \%$ \\
\hline 4 & $46.46 \%$ & $83.84 \%$ & Purpose of trip & & \\
\hline 5 & $13.13 \%$ & $96.97 \%$ & Job/study & $71.72 \%$ & $71.72 \%$ \\
\hline 6 & $3.03 \%$ & $100.00 \%$ & Shopping/free time & $28.28 \%$ & $100 \%$ \\
\hline
\end{tabular}


group characteristics. For sake of notice, route $(22.6 \%)$, weather and alternative modes to private car $(13.6 \%)$, traffic condition and departure/arrival time for transit $(11.6 \%)$ are the information type most sought; whereas web site $(32 \%)$, map (16\%) and GPS (14\%) are the main information sources. The Figure 2 shows reasons that discourage the use of transit. It should be noted that $30 \%$ of respondents consider service quality low, $24 \%$ the departure and arrival time inadequate and $16 \%$ the departure and arrival time unreliable.

\section{Latent Class Model}

The main aim of this study is, on the one hand, to understand how unreliable information influences user behaviour, and thus, how much it discourages public transport use; on the other hand, it is to assay preference heterogeneity across respondents due to both observed and unobserved effects. Only a part of the variability in the intensity of the assay can be associated with measurable socio-economic characteristics, and hence there remains a component of heterogeneity associated with these unobservable characteristics. This component can be revealed and identified by models with variable parameters, continuous distributions (mixed logit), or discrete distributions (latent class). For a more detailed description on advantages and disadvantages of both models see Green and Hensher, [35]. These models have a high capability to reproduce the individual choice behaviour and allow one better to explain the underlying individual choice mechanisms. For these tasks, we calibrated a latent class model and compared it with a traditional multinomial logit model.

Therefore, the heterogeneity across individuals is modelled with a discrete distribution, assuming that individuals are implicitly sorted in a set of classes, $C$, with class specific parameters and for each individual, a set of probabilities defined over the classes.

The choice probability of the individual $i$, among $j$ alternatives, at choice situation $t$, given that he/she is in the class $c$, is given by following equation:
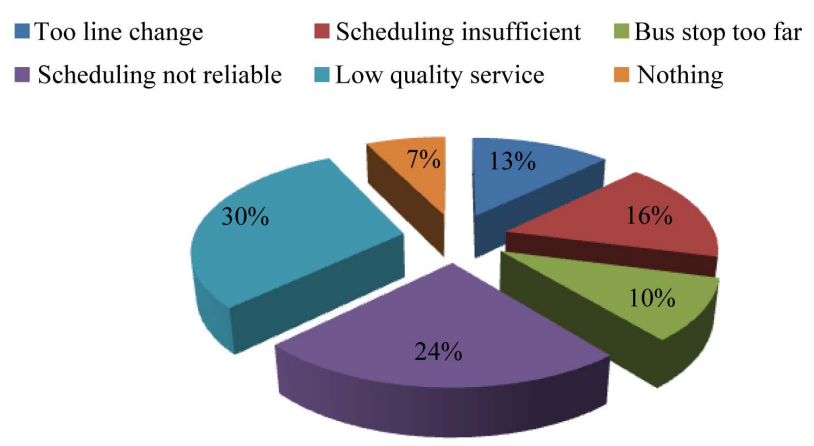

Figure 2. Reasons that disincentive the use of transit.

$$
\begin{aligned}
& \operatorname{Prob}(\text { choice } j \text { by individual } i \text { in situation } t \mid \text { class } c) \\
& =\frac{\exp \left(V_{i t, j \mid c}\right)}{\sum_{j=1}^{J_{i}} \exp \left(V_{i t, j \mid c}\right)}=P_{i t, j \mid c}
\end{aligned}
$$

where $V_{i t, j / c}$ is the systematic utility of the perceived utility $U_{i t, j / c}$ expressed as:

$$
U_{i t, j \mid c}=V_{i t, j \mid c}+\varepsilon_{i t, j \mid c}=\boldsymbol{x}_{i t, j} \boldsymbol{\beta}_{c}^{\prime}+\varepsilon_{i t, j \mid c}
$$

$\boldsymbol{x}_{i t, j}$ is a vector of $K$ attributes of choice $j$ in choice situation $t$ faced by individual $i$. $\varepsilon_{i t, j / c}$ is a random component Independently and Identically Distributed (IID) extreme value across individual, alternatives and choice situations; whereas $\boldsymbol{\beta}_{c}^{\prime}$ is the vector of class specific parameters.

Class probabilities are specified in according to the multinomial logit form:

$$
\begin{aligned}
& \operatorname{Prob}(\text { class } c \text { for individual } i) \\
& =\frac{\exp \left(\boldsymbol{\theta}_{c}^{\prime} \boldsymbol{z}_{i}\right)}{\sum_{c=1}^{C} \exp \left(\boldsymbol{\theta}_{c}^{\prime} \boldsymbol{z}_{i}\right)}=P_{i c}, c=1, \cdots, C, \theta_{c}=0
\end{aligned}
$$

where $z_{i}$ is a vector of observable characteristics (as such as, socio economic and psychometric characteristics of individual) and $\boldsymbol{\theta}_{c}$ a vector of parameters (last of which is fixed at zero). The probability that a individual $i$ makes a specific choice $j$ is expressed by:

$$
\begin{aligned}
P_{i t, j} & =\sum_{c=1}^{C} P_{i t, j \mid c} \cdot P_{i c} \\
& =\sum_{c=1}^{C} \frac{\exp \left(\boldsymbol{\theta}_{c}^{\prime} \boldsymbol{z}_{i}\right)}{\sum_{c=1}^{C} \exp \left(\boldsymbol{\theta}_{c}^{\prime} \boldsymbol{z}_{i}\right)} \frac{\exp \left(\boldsymbol{x}_{i t, j} \boldsymbol{\beta}_{c}^{\prime}\right)}{\sum_{j=1}^{J_{i}} \exp \left(\boldsymbol{x}_{i t, j} \boldsymbol{\beta}_{c}^{\prime}\right)}
\end{aligned}
$$

An issue that the analyst has to face is the choice of the number of classes, $C$. This parameter must be imposed exogenously; Train [36], suggests two criteria to assist in determining the number of classes, $C$. The former is Akaike Information Criterion AIC and the second is the Bayesian Information Criterion BIC. This latter is defined by:

$$
\begin{aligned}
& \mathrm{BIC}=2 \times \log (\text { maximized likelihood }) \\
& -(\text { no. of parameters }) \cdot \log (n)
\end{aligned}
$$

where $n$ is the number of observations.

\section{Specification of Model}

The stated preference survey on an individuated sample was carried out in order to collect data and hence, to calibrate the demand model. In our analysis, we took significant attributes into account: information cost, information inaccuracy, travel time, waiting time; socio economic characteristics: household income and daily travelled 
distance; and cut-offs relating to information cost (upper bound), information inaccuracy (upper bound), and waiting time (upper bound). The significant discrete randomly distributed parameters over classes are those relating to information inaccuracy, cut-off of the waiting time, Alternative Specific Constant ASC and household income whereas all others are non-random parameters.

Let $V_{\text {car/c }}$ be the private car utility function; $V_{\text {transit/c }}$ the public transport utility function; $C_{i}$ the daily cost in $€$ for $i=c a r$, transit; $T T_{i}$ the total daily travel time in minutes for $i=$ car, transit; $P R$ the parking research time in minutes; $W T$ the waiting time in minutes; IA the information inaccuracy in minutes; hinc $=$ decision-maker's household-income (classes 1 range less than 25,000 €; 2 range 25,000 - 50,000 €; 3 range 50,000 - 75,000 €; 4 range more than $75,000 €) ; A S C_{c a r}$ the private car specific constant; TD the daily travelled distance in $\mathrm{km}$ (classes 1 range less than $5 \mathrm{~km} ; 2$ range $5-10 \mathrm{~km} ; 3$ range $10-15$ $\mathrm{km} ; 4$ range more than $15 \mathrm{~km})$; cutoff, , cutoff WT $_{\text {, cutoff }}$ IA the cut-offs relating to cost (upper bound), information inaccuracy (upper bound), waiting time (upper bound). Cut-offs were coded by penalties dummy variables that take the values 1 if the threshold is not violated and 0 otherwise, for each decision maker; $\beta_{\text {cut,c/c }}, \beta_{\text {cut,WT/c, }}, \beta_{\text {cut.IA/c }}$ the cut-off parameters; $\beta_{c / c}, \beta_{W T / c}, \beta_{T T / c}, \beta_{I A / c}, \beta_{\text {hinc/c }}$, the parameters of the cost, of the travel time, of the waiting time of information inaccuracy and of the household income.

The utility functions of the competing alternatives are expressed as follows:

$$
\begin{aligned}
& V_{\text {car } / \mathrm{c}}=\beta_{c / c} C_{c a r}+\beta_{W T / c} P R+\beta_{T T / c} T T_{c a r} \\
&+\beta_{\text {hinc } / c} \text { hinc }+A S C_{c a r / c} \\
& V_{\text {transit } / c}= \beta_{c / c} C_{\text {transit }}+\beta_{W T / c} W T+\beta_{T T / c} T T_{\text {transit }} \\
&+\beta_{I A / c} I A+\beta_{c u t, c / c} \text { cutoff } \\
&+\beta_{c u t, W T / c} \text { cutoff } \\
& W T+\beta_{c u t, I A / c} \text { cutoff } \\
& I A
\end{aligned}
$$

All coefficients of the utility functions were achieved by a calibration process. The calibration of the latent class logit model was performed by the simulated log likelihood using the NLOGIT $^{\circledR} 4.0$ software. During the calibration process, different number of classes were tried and tested, but the best results were achieved using three classes.

\section{Outcomes of Models}

\begin{tabular}{|c|c|c|c|c|c|c|c|c|c|}
\hline \multirow{3}{*}{ Attribute } & \multirow{3}{*}{ Parameter } & \multirow{2}{*}{\multicolumn{2}{|c|}{ Multinomial Logit }} & \multicolumn{6}{|c|}{ Latent Class Logit } \\
\hline & & & & \multicolumn{2}{|c|}{ Class 1} & \multicolumn{2}{|c|}{ Class 2} & \multicolumn{2}{|c|}{ Class 3} \\
\hline & & Coeff. & t-ratio & Coeff. & t-ratio & Coeff. & t-ratio & Coeff. & t-ratio \\
\hline$C$ & $\beta_{c / c}$ & -1.656 & -5.726 & -2.365 & -7.034 & -2.365 & -7.034 & -2.365 & -7.034 \\
\hline$W T$ & $\beta_{W T / c}$ & -0.073 & -4.283 & -0.106 & -5.311 & -0.106 & -5.311 & -0.106 & -5.311 \\
\hline$T T$ & $\beta_{T T / c}$ & -0.036 & -2.141 & -0.053 & -2.690 & -0.053 & -2.690 & -0.053 & -2.690 \\
\hline cutoff $_{c}$ & $\beta_{c u t, c / c}$ & -1.324 & -7.134 & -1.287 & -5.288 & -1.287 & -5.288 & -1.287 & -5.288 \\
\hline cutoff $_{W T}$ & $\beta_{c u t, W T / c}$ & -1.058 & -5.653 & -0.485 & $-1.848^{(*)}$ & -1.644 & -2.070 & -3.170 & -5.109 \\
\hline cutoff $f_{I A}$ & $\beta_{\text {cut.IA } / c}$ & -0.638 & -3.146 & -2.295 & -6.287 & -2.295 & -6.287 & -2.295 & -6.287 \\
\hline hinc & $\beta_{\text {hinc } / \mathrm{c}}$ & +0.186 & +2.037 & +0.067 & $+0.442^{(*)}$ & +1.925 & +4.126 & +1.098 & +4.193 \\
\hline$A S A_{c a r}$ & $A S C_{c a r / c}$ & +2.142 & +2.394 & +5.853 & +5.407 & -6.564 & -3.296 & -5.941 & -2.700 \\
\hline ProbCls 1 & & - & - & +0.649 & +7.311 & & & & \\
\hline ProbCls2 & & - & - & +0.159 & +3.268 & & & & \\
\hline \multirow[t]{2}{*}{ ProbCls3 } & & - & - & +0.192 & +4.181 & & & & \\
\hline & \multicolumn{3}{|c|}{ Model Simulation } & & & & & & \\
\hline Log-likelihood (0) & \multicolumn{2}{|l|}{$L L(0)$} & -548.972 & \multicolumn{2}{|c|}{-548.972} & & & & \\
\hline Log-likelihood (B) & \multicolumn{2}{|l|}{$L L(B)$} & -415.629 & \multicolumn{2}{|c|}{-365.282} & & & & \\
\hline Chi-square [d.o.f.] & \multicolumn{2}{|l|}{$\chi^{2}[]$} & $266.666[8]$ & \multicolumn{2}{|c|}{367.381 [19] } & & & & \\
\hline Adj. pseudo $R^{2}$ & \multicolumn{2}{|l|}{$R^{2}$} & 0.242 & \multicolumn{2}{|c|}{0.334} & & & & \\
\hline Observations & \multirow[t]{2}{*}{$N$} & & 880 & \multicolumn{2}{|c|}{880} & & & & \\
\hline$B I C$ & & & - & \multicolumn{2}{|c|}{1.082} & & & & \\
\hline
\end{tabular}

The results of the calibration process of the latent class logit model are reported in Table 3, comparing them with those of the traditional multinomial logit. The latent

Table 3. Comparison between latent class logit and multinomial logit model with cut-offs.

Note: $A S A_{c a r}$ is the Alternative Specific Attribute equal to one; ${ }^{(*)}$ : non-significant parameter. 
class logit model is statistically significant, and it has a higher log-likelihood $(-365.3)$ than multinomial logit one $(-415.6)$. Further, it has a greater capability to explain the individual choice behaviour. The pseudo $R^{2}(0.334)$ is higher than multinomial logit (0.242), but the number of parameters to be estimated is greater (20) rather than nine parameters of multinomial logit, and hence it is more complex.

All parameters estimated have the correct sign and are significant, except two, the waiting time cut-off $\beta_{\text {cut,WT/1 }}$ and the household income $\beta_{\text {hinc } / 1}$, for the first class. It should be noted that cost is the most important attribute, whereas waiting time coefficient is about twice the travel time coefficient, in accordance with the scientific literature. Further, the coefficient of the information inaccuracy is the second best attribute. This shows that the decision maker gives a great importance to the reliability of the information provided and the disutility related to uncertainty of information is perceived very negatively. This aspect is also justified by opinion of respondents about the low quality of service, and often the low quality of the information provided. The survey shows that respondents meet difficulties about finding information and considering it reliable. All cut-offs are significant and have the correct sign, since the cut-off has the effect of enhancing the coefficient of the relative attribute. All class probabilities are statistically significant, highlighting the existence of heterogeneity in the estimates of parameters over the sampled population. The existence of heterogeneity is caused by Information Inaccuracy, Waiting Time cut-off, House-hold Income and Alternative Specific Constant. Furthermore, it should be noted that all other are non-random parameters.

Thus, the calibrated model suggests that heterogeneity (differences in parameters of classes) may be, in part, explained by differences in personal household income level in the information perceived (on the reliability of information) and in the perception of waiting time. Further, high values of Alternative Specific Constants over three classes suggested the analyst should take into account other attributes relevant for decision process. However, this aspect does not compromise the focus of analysis which is to understand how unreliable information influences the choice behavior and how it is a great source of heterogeneity.

Figures $\mathbf{3}$ and $\mathbf{4}$ show the plots of choice probability in term of additional information cost and information inaccuracy.

Some scenarios were constructed to show how choice probabilities change increasing cost and improving of information inaccuracy by a given percentage over the base or reference scenario. The choice probabilities are reported in Table 4. Scenario 1 is characterized by a $10 \%$ increment in information cost and a $50 \%$ improvement in information inaccuracy. Scenario 2 foresees a $20 \%$ increment in information cost and a 50\% improvement in information inaccuracy. It should be noted how a $6.7 \%$ increment in choice probabilities can be achieved increasing of $10 \%$ the information cost and improving the reliability of information provided.

The elasticity of attribute cost, information inaccuracy, travel time and waiting time provides useful information on the sensitivity of the calibrated model to the variation in a given attribute. The direct elasticity shows the effect due to a change in the value of the independent variable against the value of the dependent one. Table 5 shows the values related to the direct elasticity effect of the analyzed attributes against the probability of choosing between two alternatives (Private car, Transit), averaged over the set of observations. These data show how an increment in cost equal to $1 \%$ induces an average reduction in choice probability equal to about $3.7 \%$ for the private car and $1.5 \%$ for transit. They also highlight high cost-related demand elasticity; whereas for the attribute relating to information inaccuracy, the reduction of choice probability is about $0.52 \%$, and the demand elasticity found for the travel and waiting time is inelastic, and indeed its value is lower than one.

Finally, we tested the calibrated models on an independent data set (not used for the calibration process) made up of 11 respondents, in order to validate calibrated models. Some statistical indexes were used to test the

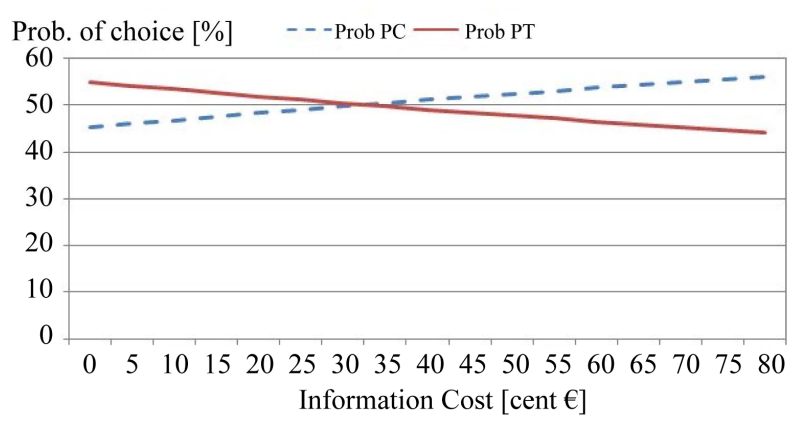

Figure 3. Probability choice in terms of information cost.

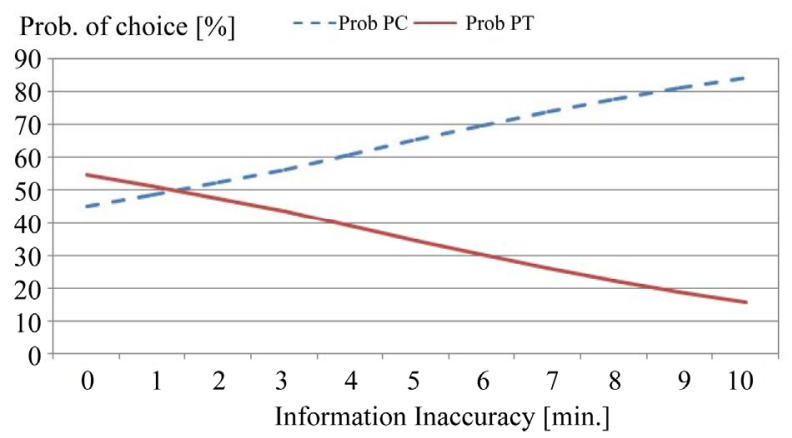

Figure 4. Probability choice in terms of information inaccuracy. 
Table 4. Choice probability in different scenarios.

\begin{tabular}{cccccc}
\hline & Scenario base (\%) & Scenario 1 (\%) & $\Delta \mathrm{P}($ Sc.1 - Sc.b) (\%) & Scenario 2 (\%) & $\Delta \mathrm{P}(\mathrm{Sc} .2-\mathrm{Sc} . \mathrm{b})(\%)$ \\
\hline Public transport & 54.83 & 61.53 & 6.70 & 57.36 & 2.53 \\
Private car & 45.17 & 38.47 & -6.70 & 42.64 & -2.53 \\
\hline
\end{tabular}

Table 5. Direct elasticity split by choice alternative.

\begin{tabular}{ccccc}
\hline Alternatives & Cost & Travel Time & Waiting Time & Information Inaccuracy \\
\hline Private Car & -3.686 & -0.344 & -0.276 & - \\
Transit & -1.509 & -0.292 & -0.231 & -0.517 \\
\hline
\end{tabular}

goodness of fit between stated and estimated choices, nominally correlation coefficient $(\mathrm{R})$, determination coefficient $\left(\mathrm{R}^{2}\right)$ and Root Mean Square Error (RMSE). Table 6 shows statistical indexes for the validation data set. The calibrated models have a good capability to simulate users' choices; in particular models with cut-offs are able to explain better the heterogeneity of users' choices.

\section{Willingness to Pay}

The Willingness to Pay (WTP) for an attribute of alternative $j$ is the ratio of the marginal utility of the attribute on the marginal utility of its cost, which in the case of linear form of utility is the ratio of the attribute coefficient on the cost coefficient.

$$
W T P=\frac{\partial V / \partial T T}{\partial V / \partial C}
$$

Table 7 shows the Willingness to Pay for each class. It should be noted that WTPs related to travel time (TT) and waiting time (WT) for the latent class model are close to multinomial logit's ones. The Willingness to Pay for information inaccuracy (IA) attribute is variable over classes and for class 1 is low $(3.6 € / \mathrm{h})$, whereas for classes 2 and 3 are about $36 € / \mathrm{h}$ and $20 € / \mathrm{h}$, respectively. This confirms the great importance given in information.

Therefore, the random parameter related to information inaccuracy is distributed in according to a discrete distribution. This implies a distribution of the WTP. An approach to achieve the entire distribution of WTP is to construct estimates of individual specific preferences deriving the conditional distribution, by using Bayes rule to find the conditional density for the random parameters (Hensher et al. [37]).

$$
\begin{gathered}
\hat{P}_{c / i}=\frac{\hat{P}_{i / c} \cdot \hat{P}_{i c}}{\sum_{c=1}^{C} \hat{P}_{i / c} \cdot \hat{P}_{i c}} \\
\hat{\boldsymbol{\beta}}_{i}=\sum_{c=1}^{C} \hat{P}_{c / i} \cdot \hat{\boldsymbol{\beta}}_{c}
\end{gathered}
$$

By followed approach we have estimated the conditional distributions of WTP related to the information inaccuracy, that is reported in Figure 5.
Table 6. Statistical indexes on validation data set.

\begin{tabular}{ccc}
\hline $\mathbf{R}$ & $\mathbf{R}^{2}$ & RMSE \\
\hline 0.69 & 0.45 & 0.225 \\
\hline
\end{tabular}

Table 7. WTPs for each class in $€ / h$.

\begin{tabular}{ccccc}
\hline \multirow{2}{*}{ WTP } & $\begin{array}{c}\text { Multinomial Logit } \\
{[\boldsymbol{€} / \mathbf{h}]}\end{array}$ & \multicolumn{3}{c}{ Latent Class [€/h] } \\
\cline { 3 - 5 } & 2.645 & 2.689 & 2.689 & 2.689 \\
\hline WT & 1.304 & 1.345 & 1.345 & 1.345 \\
TT & 8.587 & 3.653 & 35.949 & 20.499 \\
IA & & & & Class 2 \\
\hline
\end{tabular}

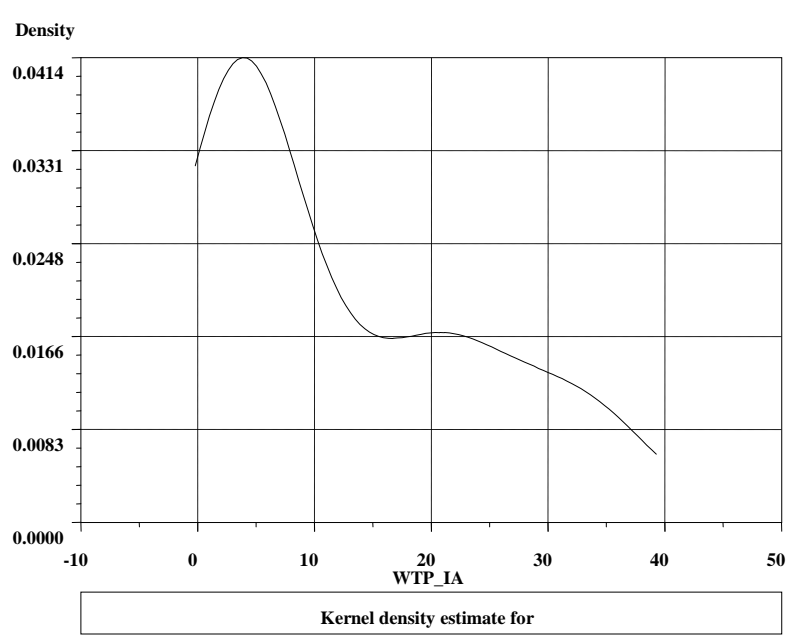

Figure 5. Conditional distributions of WTP against IA.

Table 8 shows the descriptive statistics of WTP related to the information inaccuracy. It should be noted as the mean value and the standard deviations of WTP are $12.02 € / \mathrm{h}$ and $11.39 € / \mathrm{h}$ respectively. Further, ordering WTP values, we have pointed out the trend of WTP as shown in Figure 6. Thus, the respondents have highlighted a high willingness to pay to achieve accurate and reliable information about their travel. We can affirm that the WTP for information inaccuracy is much greater than travel and waiting time WTPs. Further the perceived information is a source of heterogeneity as pointed out by 
Table 8. Descriptive statistics of WTPs.

\begin{tabular}{cc}
\hline & WTP_IA $[€ / \mathrm{h}]$ \\
\hline Mean Value & 12.02 \\
Std. Dev. Value & 11.39 \\
Min Value & 3.65 \\
Max Value & 35.82 \\
\hline
\end{tabular}

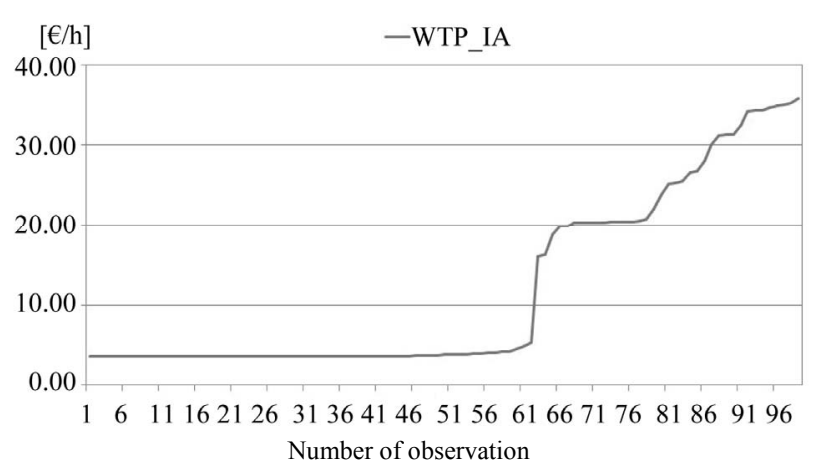

Figure 6. Trend of WTP against IA.

outcomes of calibrated models.

\section{Conclusions}

The aim of analysis is to understand how unreliable information influences user behaviour and how much it discourages public transport use. For this purpose, a Stated Preference Survey was carried out in order to know the preferences of public transport users relating to information needs and uncertainty about the information provided by Advanced Traveller Information System (ATIS). The perceived uncertainty is defined as the information inaccuracy. In our study, we have considered the difference between forecasted or scheduled waiting time at the bus stop and/or metro station provided by the ATIS, and that experienced by the user who wants to catch the bus and/or metro.

An original aspect regards the preference heterogeneity in the travel choice behaviour due to information perceived by public transport users, identifying in the decision process the unobserved heterogeneity sources. The presence of preference heterogeneity in the interviewed population sample allows one better to explain the underlying individual choice mechanisms. For this task, a latent class logit model was calibrated, taking into account attributes of cost, information inaccuracy, travel time, waiting time, and their cut-offs and comparing its results with those of the traditional multinomial logit. The latent class logit model has greater capability to explain the individual choice behaviour, but the number of parameters to be estimated is greater rather than parameters of multinomial logit, and hence it is more complex.

All parameters are statistically significant except two, parameters of waiting time cut-off and household income, for the first class. All class probabilities are statistically significant, highlighting the existence of heterogeneity in estimates of parameters over the sampled population. The presence of heterogeneity is caused by parameters Information Inaccuracy, Waiting Time cut-off, Household Income and Alternative Specific Constant whereas all other are non-random parameters.

The cost is the most important attribute, whereas the waiting time coefficient is about twice the travel time coefficient, in accordance with the scientific literature. The information inaccuracy is the second best attribute. This shows that the decision maker gives great importance to the reliability of the information provided and the disutility relating to uncertainty of information is perceived very negatively. All cut-offs are significant and have the correct sign, since the cut-off has the effect of enhancing the coefficient of the relative attribute.

Two scenarios were constructed and compared with the base scenario, showing changes in the choice probabilities, increasing the information cost and the improveing information inaccuracy. The marginal effects on transport demand have highlighted high cost-related demand elasticity; whereas for the attribute relating to information inaccuracy, the reduction in choice probability is about $0.5 \%$. This means that even a few minutes between the waiting time provided by information system and that experienced by user who wants to catch the bus and/or metro have a big weight in the user's choice. Thus the impact on the user's choice could be limited with adequate reliability of information, and in general of transit service. After, calibrated model have been tested on an independent data set to appraise prediction performance showing fairly good estimates.

Finally, the WTP for each time attribute was estimated, highlighting how population sample gives great importance in reliable information provided by ATIS. The WTP for information inaccuracy is much greater than travel and waiting time WTPs.

\section{REFERENCES}

[1] C. G. Chorus, "Traveler Response to Information," The Netherlands TRAIL Research School, Delft, 2007.

[2] M. A. Abdel-Aty, "Using Ordered Probit Modeling to Study the Effect of ATIS on Transit Ridership," Transportation Research Part C, Vol. 9, No. 4, 2001, pp. 265-277. doi:10.1016/S0968-090X(00)00037-1

[3] M. A. Abdel-Aty, et al., "Investigating Effect of Advanced Traveler Information on Commuter Tendency to Use Transit," Transportation Research Record, Vol. 1550, 1996, pp. 65-72. doi:10.3141/1550-09

[4] A. J. Khattak, et al., "Willingness to Pay for Travel Information," Transportation Research Part C, Vol. 11, No. 2, 2003, pp. 137-159. 


\section{doi:10.1016/S0968-090X(03)00005-6}

[5] E. J. E. Molin, et al., "Traveler Expectations and Willingness-to-Pay for Web-Enabled Public Transport Information Services," Transportation Research Part C, Vol. 14, No. 1, 2006, pp. 57-67. doi:10.1016/j.trc.2006.05.003

[6] A. Polydoropoulou, , et al., "Willingness to Pay for Advanced Traveler Information Systems: Smartraveller Case Study," Transportation Research Record, Vol. 1588, 1997, pp. 1-9.

[7] J. L. Adler, et al., "In-Laboratory Experiments to Investigate Driver Behavior under Advanced Traveler Information Systems," Transportation Research Part C, Vol. 2, No. 2, 1994, pp. 149-164. doi:10.1016/0968-090X(94)90006-X

[8] R. H. M. Emmerink, et al., "Effects of Information in Road Transport Networks with Recurrent Congestion," Transportation, Vol. 22, No. 1, 1995, pp. 21-53. doi:10.1007/BF01151617

[9] R. H. M. Emmerink, et al., "Variable Message Signs and Radio Traffic Information: An Integrated Empirical Analysis of Drivers' Route Choice Behaviour," Transportation Research Part A, Vol. 30, No. 2, 1996, pp. 135-153. doi:10.1016/0965-8564(95)00016-X

[10] E. Hato, et al., "Incorporating an Information Acquisition Process into a Route Choice Model with Multiple Information Sources," Transportation Research Part C, Vol. 7, No. 2, 1999, pp. 109-129. doi:10.1016/S0968-090X(99)00015-7

[11] R. Jou, et al., "Route Switching Behavior on Freeways with the Provision of Different Types of Real-Time Traffic Information," Transportation Research Part A, Vol. 39, No. 5, 2005, pp. 445-461. doi:10.1016/j.tra.2005.02.004

[12] S. Kenyon, et al., "The Value of Integrated Multimodal Traveler Information and Its Potential Contribution to Modal Change," Transportation Research Part F, Vol. 6, No. 1, 2003, pp. 1-21. doi:10.1016/S1369-8478(02)00035-9

[13] E. C. van Berkum, et al., "The Impact of Traffic Information," Ph.D. Thesis, Delft University of Technology. Delft, 1993.

[14] E. Avineri, et al., "Sensitivity to Uncertainty: Need for a Paradigm Shift," Transportation Research Records, Vol. 1854, 2003, pp. 90-98. doi:10.3141/1854-10

[15] T. A. Arentze, et al., "Information Gain. Novelty Seeking and Travel: A Model of Dynamic Activity-Travel Behavior under Conditions of Uncertainty," Transportation Research Part A, Vol. 39, No. 2-3, 2005, pp. 125-145. doi:10.1016/j.tra.2004.08.002

[16] C. G. Chorus, et al., "Travellers' Need for Information in Traffic and Transit: Results from a Web Survey," Journal of Intelligent Transportation Systems. Vol. 11, No. 2, 2007, pp. 57-67. doi:10.1080/15472450701293841

[17] K.V. Katsikopoulos, et al., "The Framing of Drivers' Route Choices When Travel Time Information Is Provided under Varying Degrees of Cognitive Load," Human Factors, Vol. 42, No. 3, 2000, pp. 470-481. doi:10.1518/001872000779698088
[18] K. V. Katsikopoulos, et al., "Risk Attitude Reversals in Drivers' Route Choice When Range of Travel Information Is Provided," Human Factors, Vol. 44, No. 3, 2002, pp. 466-473. doi:10.1518/0018720024497718

[19] K. Dziekan and K. Kottenhoff, "Dynamic at Stop RealTime Information Displays for Public Transport: Effects on Customers," Transportation Research A, Vol. 41, 2007, pp. 489-501. doi:10.1016/j.tra.2006.11.006

[20] J. Polak and P. Jones, "The Acquisition of Pre-Trip Information: A Stated Preference Approach," Transportation, Vol. 20, 1993, pp. 179-198. doi:10.1007/BF01307058

[21] P. Nijkamp, et al., "Public Transport Information Systems: An English Case Study," In: Telematics and Transport Behaviour, Springer, Heidelberg, 1996, pp. 137-157.

[22] R. G. Mishalani, et al., "The Value of Real-Time Bus Arrival Information under Various Supply and Demand Characteristics," ITS America 10th Annual Meeting, Boston, 1-4 May 2000, pp. 303-324.

[23] M. D. Hickman and N. H. M. Wilson, "Passenger Travel Time and Path Choice Implications of Real-Time Transit Information," Transportation Research C, Vol. 3, 1995, pp. 211-226. doi:10.1016/0968-090X(95)00007-6

[24] J. W. Grotenhuis, et al., "The Desired Quality of Integrated Multimodal Travel Information in Public Transport: Customer Needs for Time and Effort Savings," Transport Policy, Vol. 14, 2007, pp. 27-38. doi:10.1016/j.tranpol.2006.07.001

[25] J. Lappin, "Understanding and Predicting Traveller Response to Information. A Literature Review," 2001.

[26] S. I. J. Chien, et al., "Development of a Probabilistic Model to Optimize Disseminated Real-Time Bus Arrival Information for Pre-Trip Passengers," Journal of Advanced Transportation, Vol. 41, No. 2, 2007, pp. 195-215. doi:10.1002/atr.5670410205

[27] M. C. Tan, et al., "An Algorithm for Finding Reasonable Paths in Transit Networks," Journal of Advanced Transportation, Vol. 41, No. 3, 2007, pp. 285-305. doi:10.1002/atr.5670410305

[28] M. Lehtonen and R. Kulmala, "Benefits of Pilot Implementation of Public Transport Signal Priorities and Real-Time Passenger Information," Transportation Research Record, Vol. 1799, 2002, pp. 18-25. doi:10.3141/1799-03

[29] J. Y. K. Luk and C. Yang, "Impact of ITS Measures on Public Transport: A Case Study," Journal of Advanced Transportation, Vol. 35, No. 3, 2002, pp. 305-320. doi:10.1002/atr.5670350308

[30] P. Zito, et al., "The Effect of Advanced Traveller Information Systems on Public Transport Demand and Its Uncertainty," Transportmetrica, Vol. 7, No. 1, 2011, pp. 3143. doi:10.1080/18128600903244727

[31] K. E. Watkins, et al., "Where Is My Bus? Impact of Mobile Real-Time Information on the Perceived and Actual Wait Time of Transit Riders," Transportation Research Part A, Vol. 45, 2011, pp. 839-848. doi:10.1016/i.tra.2011.06.010

[32] J. Swait, “A Non Compensatory Choice Model Incorpo- 
rating Attribute Cutoffs," Transportation Research Part B, Vol. 35, 2001, pp. 903-928.

doi:10.1016/S0191-2615(00)00030-8

[33] G. Kocur, et al., "Guide to Forecasting Travel Demand with Direct Utility Assessment," 1982.

[34] J. de Ortúzar, et al., "Modelling Transport," 3rd edition, John Wiley \& Sons, New York, 2001.

[35] W. H. Greene and D. A. Hensher, “A Latent Class Model for Discrete Choice Analysis: Contrasts with Mixed Logit," Transportation Research Part B, Vol. 37, 2003, pp. 681698. doi:10.1016/S0191-2615(02)00046-2

[36] K. Train, "Discrete Choice Methods with Simulation," Cambridge University Press, Cambridge, 2003. doi:10.1017/CBO9780511753930

[37] D. A. Hensher, et al., "Applied Choice Analysis a Primer," Cambridge University Press, Cambridge, 2005. 